\title{
INVESTIGATION OF THE STABILITY OF SATELLITE LARGE ANGLE ATTITUDE MANOEUVRES USING NONLINEAR OPTIMIZATION METHODS
}

M.A.H. Dempster and G.M. Coupé

International Institute for Applied Systems Analysis, Laxenburg, Austria

RR-83-14

June 1983

Reprinted from Proceedings of the IFAC/ESA Symposium on Automatic Control in Space, ESTEC, Noordwyk, The Netherlands (1982) 
Research Reports, which record research conducted at IIASA, are independently reviewed before publication. However, the views and opinions they express are not necessarily those of the Institute or the National Member Organizations that support it.

Reprinted with permission from IF AC Publications.

Copyright @ 1982 IFAC Automatic Control in Space.

All rights reserved. No part of this publication may be reproduced or transmitted in any form or by any means, electronic or mechanical, including photocopy, recording, or any information storage or retrieval system, without permission in writing from the copyright holder. 


\section{PREFACE}

Research into optimization methods has long been an internationally recognized strength of System and Decision Sciences at IIASA. The research reported in this paper continues the tradition in studying the application of current numerical optimization techniques to the development of a computer-based tool for the stability analysis of general nonlinear dynamical systems.

The authors first describe mathematical models for an important aerospace engineering problem concerning the global stability of large-angle-slew attitude manoeuvres for rigid spacecraft. (Currently flying control systems perform large angle manoeuvres stably only in one plane of rotation at a time - a method that is particularly time- and energyconsuming for spacecraft on astronomical and communications missions.) The paper goes on to apply nested optimization to the Lyapunov stability analysis of the resulting systems and describes a computer-based stability analyzer currently under test.

From the methodological point of view, the nonlinear nondifferentiable controlled dynamical systems studied represent a challenge to the current level of optimization technology in spite of being posed in only six dimensions. The methods and software described are currently under development and aim in the long run to replace costly and logically inconclusive simulation studies with an exact and computationally effective designer's tool. Success in this endeavor would have significant implications for engineering and policy analysis involving dynamical systems. 


\title{
INVESTIGATION OF THE STABILITY OF SATELLITE LARGE ANGLE ATTITUDE MANOEUVRES USING NONLINEAR OPTIMIZATION METHODS
}

\author{
M. A. H. Dempster* and G. M. Coupé** \\ *Department of Mathematics, Statistics and Computing Science, Dalhousie University, Halifax, \\ B3H 4 H8 Canada and Balliol College, Oxford OX1 3BJ, UK \\ ${ }^{* *}$ European Space Agency, European Space Research and Technology Centre, Noordwijk
} The Netherlands

\begin{abstract}
Some theoretical investigations of large angle attitude manoeuvres have been based on the application of Lyapunov's stability theory. With this method Mortensen derived stable control laws for performing attitude manoeuvres with either thrusters or reaction wheels. Previous results were idealized in that they ignored the nonlinearities inherent in the operation of both types of actuators. The present paper extends the scope of this work by acknowledging the actuator nonlinearities. The approach selected is to estimate the domain of attraction (DOA) of the target equilibrium for the system corresponding to the idealized control laws constrained by the actuator nonlinearities. The DOA is estimated by searching for the largest region of asymptotic stability over a set of quadratic Lyapunov functions. This approach results from the apolication of a theorem of Lasalle and gives rise to two nested nonlinear optimization problems, the internal one of which is a constrained global problem with several local minima. The paper gives an overview of the progress made in solving problems of this complexity. Emphasis has been placed on the parametrization of quadratic Lyapunov functions and a detailed comparison of several candidate nonlinear ontimization techniques has been made for both the constrained and the unconstrained optimization problem. Areas requiring further research are identified.
\end{abstract}

Keywords. Large angle attitude manoeuvres, Lyapunov stability theory, control actuator nonlinearities, nonlinear global optimization, nested optimization procedures.

\section{INTRODUCTION}

Large angle attitude manoeurres of three axis stabilized spacecraft are currently usually performed by means of a sequence of single axis rotations. Such manoeuvres may take a relatively long time and their duration can often be considerably reduced by performing similtoneous three axis or slew manoeuvres. These manoeuvres are necessarily more complex but can be effected with moderate on-board computer power.

The stability of three axis attitude manoeuvres has been investigated quite some time ago by Mortensen $(1963,1968$ ) and Hrastar (1970), and more recently by Crouch et al (1980), using Lyapurov stability theory. These investigations have taken into account the cross-coupling of spacecraft axes during slew manoeuvres, but they have ignored the nonlinear constraints imposed on the controlled dynamical system by available attitude control actuators such as gas jets and reaction wheels.
The present paper extends the above results by taking into account the "hard" nonlinear characteristics of attitude control actuators. Numerical simulations indicate the alobal (asymptotic) stability of the resulting more realistic system and numerical exneriments are described with a domain of attraction (DOA) estimation procedure based on nested nonlinear optimization routines which could potentially prove the required sustem stability. Further research is needed to establish the DOA estimation nrocedure and the problems which must be solved are precisely identified. In this regard, it should be noted that numerical DOA estimation to date provides the best hope of proof of global stability of satellite large angle slew attitude control systems. Indeed, cascaded nonlinearities in several dimensions -- as incorporated in the realistic reaction wheel control system defined below -- are currently beyond the reach of frenuency domain stability analysis techniques. Moreover, simulation studies can never establish stability properties beyond doubt -- even at 
immense computational cost.

\section{MATHEMATICAL REPRESENTATION OF} SLEW MANOEUVRES

The control systems obtained by Mortensen (1963) and Hrastar (1970) for three axis manoeuvres have been reformulated by Dempster et al (1979) and Coupé (1980) in order to have a manageable mathematical representation for their extension to include actuator nonlinearities. Two cases have been distinguished: gas jet controlled manoeuvres and reaction wheel controlled manoeuvres.

Gas jet control. When the spacecraft attitude is controlled by means of extemal torques the governing system equations are

$$
\begin{array}{ll}
J \dot{\omega}=u+s(\omega) J \omega & \text { (dynamics) } \\
u=-k \omega-k r_{0}^{-3} r & \text { (control law) } \\
\dot{q}=\frac{1}{2} s(\omega) q+\frac{1}{2} q_{0} \omega & \text { (kinematics) } \\
\dot{q}_{0}=-\frac{1}{2} \omega^{\prime} q & \\
r=f_{0} q-q_{0} f+s(q) f & \text { (attitude err } \\
r_{0}=f_{0} q_{0}+f^{\prime} q &
\end{array}
$$

where:

$$
\begin{aligned}
& \text { J - spacecraft inertia matrix } \\
& \text { S(•) - matrix representation of vector } \\
& \text { product } \\
& \text { w - spacecraft inertial angular velocity } \\
& \text { u - external control torque vector } \\
& \text { K - diagonal matrix of rate gains } \\
& \text { k - scalar position gain } \\
& \left(q, q_{0}\right) \text { - body quaternion } \\
& \left(f, f_{0}\right) \text { - target quatemion } \\
& \left(r, r_{0}\right) \text { - emror quaternion }
\end{aligned}
$$$$
\text { (all in inertial space) }
$$

prime denotes transpose and dot denotes time derivative.

In this case the feedback control law (2) with nonlinearly adaptive position gain $\mathrm{k} / \mathrm{r}^{3}$ guarantees that the spacecraft may be controlled from any initial conditions for $\omega$ and $r$ to a unique final state $\omega=0$, $r=0, r_{0}=1$ provided that the control is unconstrained by actuator nonlinearities. This implies that the dynamical system (1)-(6) is globalzy asymototically stable or asymptotically stable in the Zarge.
Reaction wheet control. The equations for the gas jet case are still valid with the exception of the dynamics which must be modified for momentum exchange devices as

$j \dot{\omega}=u+s(\omega) A_{b s} h_{s}$

where:

$h_{s}$ - the constant inertial spacecraft angular momentum vector

$A_{b s}$ - the transformation matrix from inertial space to body coordinates

$A_{b s}=\left(2 q_{0}^{2}-1\right) I_{3}+2 q q^{\prime}+2 q_{0} s(q)$

$I_{3}-3 \times 3$ identity matrix.

For the qas jet case Mortensen (1968) also analyzed the stability of a feedback control law with rate gains the same as (2) but with simple nroportional position gain $k$. It has been shown by Dempster et al (1979) and Coupé (1980) that in the absence of actuator nonlinearities both control laws proposed by Mortensen can be stably implemented by either qas jets or reaction wheels. For this purpose it suffices to take the inertial coordinate frame as the target frame, so that current error and body quaternions coincide. (For simplicity we identify attitude error and body attitude below.) The Lyapunov function $V$ aiven by

$v\left(\omega, q, q_{0}\right):=\frac{1}{2} \omega^{\prime} J \omega+k q^{\prime} q / q_{0}^{2}$

is used to prove the global asymptotic stability of the present systems, but it should be noted that this Lyapunov function is easily modified to establish the stability of other control laws, ef. Mortensen (1968).

Gibbs vector representation. In the previous subsections the spacecraft kinematics has been represented by means of quaternions because they provide the simplest attitude representation. However in view of the nonlinear optimization procedures envisaged, the extra dimension and algebraic constraint of the quaternions is cumbersome. In order to avoid these somewhat artificial difficulties, the quaternion attitude representation can be replaced by the Gibbs vector representation defined in terms of the quaternion as

$$
\mathrm{p}:=\mathrm{q} / \mathrm{q}_{0} \text {. }
$$

One finds

$\dot{p}=\frac{1}{2}\left[I_{3}-S(p)+p p^{\prime}\right] \omega$

and

$A_{b s}(p)=\left(1+p^{\prime} p\right)^{-1}\left[\left(1-p^{\prime} p\right) I_{3}+2 p p^{\prime}+2 S(p)\right]$

instead of the kinematic equations (3), (4) 
and (8) in terms of quaternion, and the control law becomes

$u=-K \omega-k\left(1+p^{\prime} p\right) p$.

Expression (12) obviates the need to explic$i t l y$ consider differential equations for wheel momenta -- thereby enlarging the dimension of the staice space from 6 to 9 -as was done by Hrastar (1970).

Control actuator modeling. The control law proposed above is idealized and as such does not take into account the constraints on control torques imposed by real control actuators. In order to model real actuators the control law (13) must be modified to agree with the actual torque characteristics of the envisaged actuator. To this end, the control actuator input signal is defined to be

$s:=K \omega+k\left(1+p^{\prime} p\right) p$.

For gas jets the control law must take into account that a set of thrusters can in general produce torques of constant magnitude and varying sign. Thus (ignoring thrust rise time) the control torque $u$ is modified for thrusters to be

$u:=-T_{\max } \operatorname{sgn}(s) \quad$ (gas jets)

with:

$T_{\max }$ - maximum torque level

$\operatorname{sgn}(\cdot)-3$-vector of simple sign functions.

Each of the three sign functions (defined to be 0 at 0 ) operates on the appropriate coordinate of the controller inout signal to produce the gas jet torque $\pm T_{\max }$ for the corresponding thruster pairs.

For momentum exchange control it should be noted that reaction wheels can ideally produce torques continuously between zero and a maximum level $T_{\max }$, but to prevent

breakup they must cease to produce control torque by free spinning when the wheel speeds reach some operational limit $\omega_{\max }^{W}$.

Therefore, the reaction wheel control law can be represented as the following cascade of "hard" nonlinear functions

$u:=-\operatorname{sat}(s) h\left(\omega^{W}, s\right) \quad$ (reaction wheels)

where:

$\operatorname{sat}(\cdot)$ - $3 \times 3$ diagonal matrix of saturation functions proportional to their arguments between $\pm T_{\max }$

$h(\cdot, \cdot)$ - 3-vector of $0-1$ valued functions $h_{i}$ representing wheel motor shutdown and restart.

Each of the coordinate saturation functions operates on the appropriate coordinate of the controller input signal to produce the idealized reaction wheel torque. Each coordinate of the $h$ function is of the form

$$
\begin{array}{r}
h_{i}\left(\omega_{i}^{w}, s_{i}\right):=\max \left\{\operatorname{win}\left(\omega_{i}^{W}\right), 1-H\left(s_{i} \omega_{i}^{W}\right)\right\}, \\
i=1,2,3
\end{array}
$$

with

win(·) - window function equalling 1 for $-\omega_{\max }^{1}<\omega_{i}^{W}<\omega_{\max }^{W}$ and 0 elsewhere

$\omega_{i}^{w} \quad-i^{\text {th }}$ wheel speed, $i=1,2,3$

$\mathrm{H}(\cdot)$ - Heaviside function equalling 1 for nonnegative arguments and 0 els ewhere.

The window function in (17) serves to limit idealized wheel speed to within $\pm \omega_{\max }^{W}$, while one minus the Heaviside function calls for restart of a free spinning wheel in the opnosite direction of rotation. The effect of the maximum is to allow wheel torque output when either (or both) of these condi-

tions hold. The wheel speeds $\omega^{W}$ are obtained from

$J^{W} \omega^{W}=h_{b}-\left(J+J^{W}\right) w$

where:

J - inertia matrix of the spacecraft with locked wheels

$J^{W}-3 \times 3$ matrix of wheel inertias

and the angutar momentum vector $h_{b}$ in body coordinates is related to the constant inertial angular momentum vector $h_{s}$ as before by

$h_{b}:=A_{b s}(p) h_{s}$.

If a slew manoeuvre is begun from rest, the vehicle angular momentum is intially zero and the system angular momentum represented by $h_{s}$ must be stored in the spinning reaction wheels. (In practice, auxilliary thrusters are typically used to dump system momentum from time to time in the course of a mission.)

For reaction wheel control, the newly defined control law is necessarily asymptotically stable for states where the nonlinear actuator constraints do not onerate, i.e. in a sufficiently small neighbourhood of the target equilibrium point. For both types of control however there may be states from which the above control laws will not transfer the system to the target equilibrium point. In this case, the new slew control laws could not be imnlemented with real gas jets or reaction wheel actuators to stably perform azz large angle 
attitude manoeuvres.

Two related problems must therefore now be addressed. The first one is to estimate the set of initial conditions from which the new controllers transfer the system asymptotically to the equilibrium point, i.e. the systems' domain of attraction. Secondly, to show that these domains of attraction are the whole state space, i.e. to demonstrate global asymptotic stability of the new systems.

For all numerical experiments (and in the sequel) the attitude control systems described above were represented in their most simple general form as a nonlinear 6-vector differential equation

$\dot{x}=f(x)$

where $x^{\prime}:=\left(\omega^{\prime}, p^{\prime}\right)$ and $f(x)$ includes the nonlinear control laws incorporating the actuator constraints. The equilibrium point $x=0$ corresponds to the spacecraft at rest at the target attitude and $f(0)=0$. The corresponding control system diagrams are depicted in Figs. 1 and 2 .

\section{ATTITUDE CONTROL SYSTEM SIMULATION}

As noted above, the objective of the simulations performed was to investigate -- in a preliminary way -- the stability boundaries of the proposed controllers of a hypothetical spacecraft executing 3 -axis slew manoeuvres. For detailed numerical parameter values used in the mathematical models see Dempster et al (1979), \$1.5. It is sufficient here to note that all numerical experiments were conducted with system parameters corresponding to a large vehicle of the NASA Orbiting Astronomical Observatory $(\mathrm{OAO})$ type. The control systems considered were:

1. the gas jet system

2. the reaction wheel system with zero initial spacecraft momenta

3. the reaction wheel system with Zarge initial spacecraft momenta.

It was assumed in all cases that the initial angular velocities of the vehicle were zero, and that the desired rotation angle was as close to $180^{\circ}$ (reverse-point manoeuvre) as possible. A unit eigenaxis and an angle of $179^{\circ}$ were arbitrarily chosen to define the initial Gibb's vector representation $p(0)$ of the inertial attitude error of the body relative to the target inertial body attitude. Simulations of all the 8 combinations of sign of the components of $p(0)$ were made to verify the stability of the proposed controllers. All simulations were run on a PDP 11/45 computer with floatingpoint hardware running under the IAS operating system. The simulation program used was Oxford Systems Associates' Extended System Modelling Program (ESMP) which is a general-purpose block-oriented simulator allowing for extensive user interaction.

Gas jet control. For the gas jet system, a11 8 simulations of $179^{\circ}$ slew manoeuvres -from each orthant of the attitude state space -- were accomplished stably with appropriate controller parameters. A typical screen output is shown in Fig. 3.

Reaction wheel control. A similar statement applies to the reaction wheel system, for which periods of coasting with one or more speed limited reaction wheels spinning freely were noted. However, for the reaction wheel system with high initial system momentum ( $24 \mathrm{Nms}$ ), instability was encountered from one of the 8 initial attitudes. Even with drastically reduced rate gains (35 to 7 ) in the control input signal, stability for only $168^{\circ}$ slew manoeuvres appeared possible from this position and even then attitude error tended to decay rather slowly, with dying oscillations still visible after 5000 seconds of manoeuvre time. All the reaction wheel system simulations described were with parameters corresponding to wet 1 ubricated reaction wheels with maximum speeds of $40 \pi \mathrm{rad} / \mathrm{s}$. The final simulations were for a reaction whee 1 system equipped with magnetic bearing wheels with maximum speeds of $300 \pi \mathrm{rad} / \mathrm{s}$, very high initial system momentum (100 vs. $24 \mathrm{Nms}$ ) and adjusted controller parameters. Stable $179^{\circ}$ slew manoeuvres were possible for this system from the attitude error previously inducing instability -- with only a single wheel reaching its speed limit once, see Fig. 4. Controller parameter tuning could however clearly be improved.

In summary, the simulation experiments indicated that -- with suitable parametrization -- both new large angle attitude control systems are globally stable. (A more detailed discussion may be found in Dempster (1980a) and Dempster et al (1979).)

\section{ESTIMATION OF THE DOMAIN OF ATTRACTION}

The thrust of the present research is nevertheless to ultimately replace computationally expensive and scientifically inconelusive stability analys is of dynamical systems by simulation with a computationally efficient and decisive procedure. Hence there is a need to estimate the domain of attraction of the equilibrium point $x=0$ of the system (20) by maximizing the region of asymptotic stability corresponding to a particular type of Lyapunov function over the parameters defining the function (and possibly the control law).

Consider the autonomous nonlinear dynamical system given by the vector differential equation

$\dot{x}=f(x)$

where $x$ is an $n$-vector in the (Euclidean) state space $\mathbb{R}^{n}$ of the system, $f$ is a 
continuously differentiable $n$-vector valued function of an $n$-vector argument $\left(f: \mathbb{R}^{n} \rightarrow \mathbb{R}^{n}\right.$, $f \varepsilon c^{1}$ ) with $f(0)=0$. The origin 0 of the state space is an asymptotically stable equilibrium point of the system (21) with respect to a domain $\Omega$ in state space if, and only if, for all initial points $x(0):=x_{0}$ in $\Omega$ the corresponding solution trajectories of (21) tend to 0 as $t$ tends to infinity. That is, the system eventually returns to equilibrium from any initial point in the domain $\Omega$, which is a region of asymptotic stabizity (RAS) of 0 for the system. The maximal RAS of 0 is the domain of attraction (DOA) of 0 for the system. The distinction between the two concepts is that an RAS may be defined in terms of a Lyapunov function by means of a theorem of Lasalle, while the DOA is rather an absolute system property.

LasalZe's theorem. In order to apply Lyapunov's second or direct method to the identification of an RAS for the system (21) an appropriate definition of an "energylike" function is needed. A real valued function $V$ defined on a domain $\Omega$ of state space $(V: \Omega \rightarrow \mathbb{R})$ is positive definite on $\Omega$ if, and only if, $V(x)>0$ for all nonzero state vectors $x$ in $\Omega$ and $V(0)=0$. Such a function $V$ is negative definite if, and only if, $-V$ is positive definite. The following theorem, due to Lasalle, gives a method for using a more closely specified "energy-like" Lyapunov function to identify regions of asymptotic stability, see Lasalle \& Lefschetz (1961). Chapter 2, $\$ 9$ and Rouche et al (1977).

THEOREM: Let $\Omega$ be an open domain in $\mathbb{I R}^{n}$ and let $V$ be a real valued function of an n-vector argument which is continuously differentiable and positive definite. Consider the open region

$\Omega^{*}=\{x \varepsilon \Omega: V(x)<v\}$

inside the contour of $V$ at level $v>0$. If $\Omega^{*}$ is a bounded set -- i.e. $V$ has closed curve contours -- and the time derivative $\dot{V}$, given by

$\dot{V}(x):=\nabla V(x) f(x)$,

of $V$ is negative definite on $\Omega^{*}$, then 0 is an asymptotically stable equilibrium point of the dynamical system (21) and $\Omega$ * $i s$ an RAS of 0 .

In expression (23) $\quad \nabla V(x)$ denotes the gradient of the Lyapunov function $V$, i.e., the $n$-row vector of partial derivatives of $\checkmark$ given by

$\nabla V(x):=\left(\partial V(x) / \partial x_{1} \ldots \partial V(x) / \partial x_{n}\right)$.

If the dynamical system (21) is not gzobazzy (asymptotically) stable Lasalle's theorem implies that there exists a state vector $x$ at which $\dot{V}(x)=\nabla V(x) f(x)>0$ - that is, the time rate of change of "energy" is increasing. Since the Lyapunov function $V$ is assumed continuously differentiable, it follows that there must exist a nonlinear manifoid

$M:=\left\{x \in \mathbb{R}^{n}: \dot{V}(x)=0, x \neq 0\right\}$

on which $\dot{V}$ vanishes. This manifold is in general multibranched and it is a practically important open mathematical problem to completely characterize the manifold $M$ for suitable classes of Lyapunov functions and dynamical systems relevant to aerospace and other applications, ef. Shields(1973).

It follows from the above that a maximal RAS $\Omega^{*}$ corresnonding to a specific Lyanunov function $\dot{V}$ may be generated by isolating the appropriate Zocal solution(s) $x^{*}$ of the nonlinear programming problem

$\min _{x} V(x)$ subject to $\dot{V}(x)=0$.

Note that since $V$ and $\dot{V}$ vanish at 0 , 0 is a triviat solution of (26). Thus we actually seek a solution $x^{*}$ of the nonlinear programming problem

$\min _{x} V(x)$ subject to $\dot{V}(x)=0, x \neq 0$

or, using (25), min $\operatorname{m\in M}_{\mathbb{M}} V(x)$. In general the discrete solution set of the programme (27) contains one or more radially symmetric pairs, see Shields \& Storey (1975), and the problem has zocal (up to first order) solutions which are not global. In this paper, methods are devised for both eliminating the trivial solution of problem (26) at the origin and for locating a global, rather than a local, solution of the programme (27). For different Lyabunov functions the RAS's defined by solving the ontimization problem (27) will in general differ.

Maximizing the RAS. In order to obtain a maximal estimate of the DOA of the dynamical system (21) using Lasalle's theorem, we may consider a parametric class of Lyapunov functions, solve the problem (27) for each of them, and choose the "largest" with respect to a suitable measure of the size of the candidate regions of asymptotic stability corresponding to the Lyapunov functions chosen at each step. Consider a parametric class of Lyapunov functions

$V:=\left\{V_{z}\right\} z \varepsilon Z$

depending on a parameter vector $z$ in a set $Z$ and denote the optimal value of the programme (27) for the Lyapunov function parametrized by $z$ by

$v_{z}^{*}:=v_{z}\left(x^{*}\right)$.

To obtain the best estimate of the DOA using Lyapunov functions from the class $V$ we must solve the nonlinear (unconstrained) 
programming problem

$\max _{z \in Z} F\left(V_{z}^{\star}, z\right)$,

where $F$ is a suitable measure of the size of the maximal RAS corresponding to a specific Lyapunov function given by the solution to (27).

Thus, combining the problems (27) and (30), we see that in order to obtain a maximal estimate for the DOA of the dynamical system (21) using a given class of Lyapunov functions, we must solve the difficult nested nonlinear optimization problem

$\max _{z \in Z} F\left(\max { }_{x \in M} V_{z}(x), z\right)$

being careful to isolate the global solution of the inner loop (RAS) optimization problem (27).

\section{QUADRATIC LYAPUNOV FUNCTIONS AND THEIR PARAMETRIZATION}

Of the three types of computable Lyapunov function classes treated in the literature:-

1. Quadratic (Rodden, 1965; Weissenberger, 1969; Geiss et al, 1971; Davison \& Kurak, 1971 ; Shields \& Storey, 1975)

2. Polynomial (Zubov, 1955)

3. Piecewise Linear (Rosenbrock, 1962)

quadratic Lyapunov functions have proven the most reliable to date. Members of this class have been used to obtain the stability of the control laws in Mortensen (1963, 1968). The practical advantages of employing quadratic Lyapunov functions in numerical stability studies are twofold.

RAS size. The first is that a constant value of the Lyapunov function $V_{p}(x)$ given by the quadratic form $X ' P x$-- and in particular its maximal RAS boundary -- represents a hyperezzipse in state space. Hence it is easy to visualize and its "size", i.e. a monotone function of its hypervolume, given at a (global) optimum of the inner loop program (27) by

$h(P):=n \log V_{P}^{*}-\log \operatorname{det} P$

where $V_{p}^{*}$ denotes the corresponding optimal value, is easy to compute.

Lyapunov's matrix equation. Secondly, quadratic forms are easily generated. The method employed in this paper is to select an arbitrary negative definite matrix -0 and then solve the Lyopunov matrix equation

$A^{\prime} P+P A=-Q$

for the kernel $P$ of the quadratic form. To see how this equation arises, consider the dynamical system (21) written in first order Taylor series expansion as

$\dot{x}=A x+g(x)$

where the $n \times n$ matrix $A:=\nabla f(0)$ and $g$ contains second and higher order terms. Neglecting $g$ in (35) and computing $\dot{v}$ directly yields

$V(x)=x^{\prime}\left(A^{\prime} P+P A\right) x:=-x^{\prime} Q x$.

Hence it follows from Lasalle's theorem that the linearization about the origin of the nonlinear system (21) is globally asymptotically stable if, and only if, Q in (36) is positive definite. To generate by solving (34) a positive definite matrix $P(Q)$, given a positive definite matrix $Q$, an $O\left(n^{3}\right)$ iterative algorithm due to Smith (1971) is available. For a brief discussion of alternatives see Dempster et al (1979), Appendix I.2.

Parametrization of positive definite matrices. The best technique for generating positive definite matrices 0 was utilized in an earlier (small angle attitude control) study by Geiss et az (1971) based on Murnaghan (1962). It is well known that all real symmetric matrices are orthogonally similar to a diagonal matrix, whose entries are its eigenvalues, and thus that all positive definite matrices are orthogonally similar to a diagonal matrix with positive diagonal elements. Hence the parametrization of all $n \times n$ positive definite matrices may be effected in the required $n(n+1) / 2$ parameters by combining a parametrization of the group of orthogonal matrices with the $n$ diagonal elements of a diagonal matrix in the form

$Q(z):=G^{\prime}(\theta, \emptyset) \wedge G(\theta, \emptyset)$

where the row vector $z^{\prime}:=\left(\lambda^{\prime}, \theta^{\prime}, \emptyset^{\prime}\right)$, $\Lambda:=\operatorname{diag}\left(\lambda_{1}, \ldots, \lambda_{n}\right)$ and $G$ is an orthogonal matrix defined by an $(n-1)(n-2) / 2$ vector $\theta$ and an $(n-1)$ - vector $\emptyset$ (see Dempster et al, 1979, Appendix I.1, for details). The advantage of this parametrization over other possible, but badly behaved, parametrizations in the same number of parameters is that with it separate adjustment of the lengths and orientations of the principal axes of the hyperellipsoidal Lyapunor function contours is possible.

We are now in a position to define precisely the objective function $f: \mathbb{R}_{m} \rightarrow \mathbb{R}$, $z \mapsto F(z)$ of the unconstrained problem (30) as

$F(z):=\operatorname{hoPoQ}(z)$.

\section{THE DOMATT SYSTEM}

Fig. 5 outlines the structure of an algorithm to estimate the domain of attraction of an equilibrium point of a general dynamical 
system based on the above theory. There are two parts to this algorithm. For any given quadratic Lyapunov function we wish to find the maximum region of asymptotic stability (RAS). A measure of the size of this region estimates the size of the domain of attraction of the equilibrium point relative to the given Lyapunov function. The RAS problem is formulated as a nonlinear programming problem which forms the inner loop of the algorithm. The second part, or outer loop, of the algorithm seeks the quadratic Lyapunov function which yields the largest RAS, and hence the optimal estimate of the domain of attraction. Again this is formulated as a nonlinear programing problem. A third optimization could be considered, but is not dealt with in this paper, namely the further maximization of the domain of attraction of the system (20) with respect to the gain parameters $k$ and $k$ used to define the new attitude control laws.

The (closed 10op) dynamical systems generated by both new controllers are of the form

$\dot{x}=\tilde{f}(x)+\tilde{g}(x)$,

where $\tilde{f}$ is an analytic function of the state vector $x$ and $\tilde{g}$ is a discontinuous, piecewise continuously differentiable function. The theory of the previous two sections on the other hand applies only to dynamical systems whose right hand sides are continuously differentiable. Unfortunately, an analytical theory of piecewise quadratic Lyapunov functions for discontinuous systems is well developed only for the case of a unidimensional discontinuity, see Weissenberger $(1965,1969)$ and Dempster et at (1979). For both attitude control systems however the discontinuous or nondifferentiable points of $\tilde{g}$ are explicitly known and few in number. It is therefore possible to provide continuously differentiable arctangent approximations to the discontinuous/ nondifferentiable functions, although these are extremely complicated, see Dempster et at (1979), Appendix IV, for details.

The key to a successful procedure for estimating the domain of attraction lies in the choice of algorithms for performing the two nonlinear optimizations described above. It was decided that the latest proven constrained and unconstrained techniques of classical type afforded the best chance of efficient optimization calculations. In particular, for the relatively few nondifferentiable points in the system dynamics due to sign (gas jet) and to saturation, window and Heaviside (reaction wheel) function modelling of control actuators, infant (and largely inefficient first order) nondifferentiable optimization techniques were not deemed worth the computational overheads. (In this regard see Lemarechal (1979b) who shows that classical methods can be effective in such situations.) Instead smooth (and other suitable) approximations to the baseline system control actuator nonlinearities have been utilized. The selection, specifi- cation, implementation and testing of the optimization routines used in the method was carefully addressed in Dempster et al (1979) where basic requirements for numerical optimization algorithms in this context were set out. Two alternative algorithms were proposed for the outer loop -- the Powell (1964) conjugate direction method, and the BFGS quasi-Newton (or variable metric) method (see, e.g. Adby \& Dempster, 1974) and one for the inner loop -- the Powe11-Han projected Lagrangian algorithm, Powel1 (1977, 1978). The performance of these algorithms was evaluated on a number of carefully specified test problems (details of which are to be found in Appendix II to Dempster et al, 1979).

For computation using the Powe11-Han algorithm, the inner loop programme (27) is replaced by the analytically equivalent problem

$V *(P):=\min _{x} x^{\prime} P x$

\section{s.t. $f(x)^{\prime} P x p(x) \geqq 0$}

where $p$ is a nonnegative radially decreasing function with a single pole at the origin of at least the same order as the zero at the origin of $f(x)^{\prime} P x$. Multiplication of the equation constraint of (27) by $p$ removes the trivial solution of (26) at 0 , while use of the inequality appears to result in improved performance of the Powel1-Han algorithm.

of course the Powell-Han routine is only capable of finding Zocal optima of (40) corresponding to solutions on various branches of the manifold

$M=\left\{x \in \mathbb{R}^{n}: f(x)^{\prime} P x=0\right\}$. In general several local minima exist (in radially symmetric pairs) and the global solution must be selected from these as yielding the smallest value of $V$. As the outer loop algorithm varies the positive definite matrix $Q$, the Lyapunov function kernel $P$ is deformed via the Lyapunov matrix equation and the branches of the manifold $M$ move in a manner which makes it impossible to predict on which branch the global solution lies. While a small change in $Q$ may move the manifold $M$ by only a small amount, the global solution may, as a result, jump to a different branch of $M$. This difficulty may be overcome by keeping track of all candidate local optima as Q varies, and by choosing at each outer loop function evaluation the global inner loop optimum from this set.

In the computer implementation of the DOA estimation algorithm an initial set $X_{0}$ of state space starting points for the PowellHan algorithm is supplied by the user. At each outer 10op function evaluation $\&$, the result of every successful inner loop estimization is added to the current set $x_{l}$, while all other elements of $x_{\ell}$ in a 
neighbourhood of this local optimum are deleted in order to prevent unlimited growth of the set $X$. In numerical experiments, it has been observed that when the manifold $M$ moves slowly with changes in $Q$, each local solution of (40) replaces the starting point which led the Powell-Han algorithm to it. (In the code, the number of starting points may be limited by the user.)

Since at the outer loop optimum there are usually multiple global solutions to the inner loop problem (27), the outer loop optimum is often located when two or more inner loop solutions of (40) (other than radially symmetric pairs) yield the same value of $\mathrm{V}$.

The algorithm outlined in Fig. 5 and incorporating the optimization routines discussed above is implemented as the structured FORTRAN code DOMATT. The general design principles and skeletal outline of the DOMATT system as a piece of applications software, together with the module structure of DOMATT'S FORTRAN code -- including the Atomic Energy Research Establishment, Harwe11, U.K. optimization routines VAO4A, VA13AD, VFO2AD and POWHAN -- may be found in Dempster (1980a,b) with more details in Dempster et al (1979). A software manual is also available from the authors.

\section{NUMERICAL EXPERIMENTS}

Dynamical system test problems. The DOMATT code has been tested in a preliminary way on 8 specially chosen test problems (see Dempster et al, 1979, Appendix III, for their specification). The criteria used in selecting these test problems were as follows:-

1. To include at least one system to which the solutions of the RAS problem and the DOA are known analytically.

2. To include a low order system of gas jet type as a simple test of the performance of the DOMATT system in the presence of "hard" nonlinearities.

3. To grade the state space dimensions of the test problems (2 through 5) in such a way as to give some indication of the increase in computation time with increase in problem complexity.

The computations were performed to an accuracy of $10^{-3}$ by an ICL2980 computer running under the VME/B operating system.

In the experiments to date the DOMATT code solved all 2-dimensional problems to the required accuracy except the jet-type problem involving a "hard" sign function nonlinearity. The outside bound error termination difficulties encountered with the Powe11-Han algorithm on this problem were exacerbated on the higher dimensional "high curvature problem" -- a 3-dimensional cubic model of a servo-mechanism. The remaining 3-dimensional problems and the 4-dimensional problem have so far not been solved to termination. It is not clear to what extent further algorithmic tuning could reduce computation times, al though there was some indication on the largest test problem that the inner 1000 Powel1-Han algorithm was taking prematurely small steps. More importantly, relatively slow progress was experienced at the outer loop by the Powe11 ' 64 conjugate direction method. If (approximate) gradient information from perturbation analys is of the inner loop problem (27) were available, faster outer loop progress could be expected with the BFGS quasi-Newton method on the basis of comparative tests of the two methods performed in the study and general exnerience, see e.g. Adby \& Dempster (1974). However, it is not clear what difficulties would be caused by nondifferentiability of the outer loop RAS measure due to multiple global solutions of the inner loop problem. This remains a topic for future research to be discussed further below. An analysis of the average time per outer loop - - i.e. candidate quadratic Lyapunov -- function evaluation, showed this average inner loop run time to a global optimum to increase very reasonably with state space dimension.

Attitude control systems. In the preliminary numerical experiments with the DOMATT code a gradient evaluation in a specified neighbourhood of a control actuator nondifferentiability was replaced by a median sub- or supergradient value, while in such a neighbourhood of a discontinuity a gradient evaluation was replaced by a specified large number of appropriate sign. Given the difficulties uncovered in the anplication of the DOMATT code to the eight test continuously differentiable problems, it was not expected that the application to the nondifferentiable attitude control systems would prove very successful without a deeper understanding of the problems experienced by the inner loop optimization routine. Thus, while both systems have been coded, the DOMATT code has so far been applied in a preliminary way only to the gas jet system. The gas jet system was presented to DOMATT with the intention of finding at least a crude DOA estimate. The first such run processed 66 inner loop optimizations -- i.e. 66 candidate Lyapunov functions -- in 30s. This is a suspiciously rapid average rate when compared to such rates for the lower dimensional test problems. In fact, in every case the inner loop global solution was found to lie in state space within the specified tolerance of the origin. When similar near zero inner loop solutions were found with the test problems, a clear contrast emerged between inner loop optimizations finding a genuine solution and those finding the trivial solution at the origin. Since the completion of the preliminary experiments, the theoretically effective nonlinear constraint rescaling technique which removes the trivial solution to the inner loop constraint 
equation at the origin -- described above in connection with the numerical inner loop problem (40) -- has been developed. The application of this technique to the gas jet system is a first priority of research currently in progress.

\section{CONCLUSIONS}

Summary. Two large angle attitude controllers, a gas jet mass expulsion system (external torque) and a reaction wheel momentum exchange system (internal torque), were modelled with respect to a new minimal dimension representation of satellite body rate dynamics, using Gibb's vector kinematics and the Mortensen '63 slew control 1aw. The models account for the most important nonlinearities in the control actuators and are known to be globally asymptotically stable when these constraints are not imposed. Both systems were extensively simulated and the simulation studies indicate their globally stability in spite of the realistic nonlinearities in the control actuator modelling. Moreover, these studies have demonstrated that notwithstanding their mathematical singularity at $180^{\circ}$ Gibb's vectors can be used to study $180^{\circ}$ reverse points to within the accuracy of a fine pointing control system.

Lasalle's theorem has been interpreted for quadratic Lyapunov functions as a basis for an inner loop optimization procedure for estimating the region of asymptotic stability (RAS) of dynamical systems of the form $\dot{x}=f(x)$. A well behaved parametrization of quadratic Lyapunov functions has been taken so that the optimal Lyapunov function (that producing the largest RAS) can be found by an outer loop optimization. Thus a procedure for estimating the domain of attraction (DOA) of an equilibrium point of a (controlled) dynamical system has been devised which implements the latest optimization techniques in the quadratic Lyapunov function approach to DOA estimation. The procedure has been incorporated in a modular FORTRAN computer code DOMATT written to advanced modern software standards.

In order to test the DOMATT code, tune algorithm parameters and gain computational experience with the method, a carefully designed set of dynamical system test problems were run. Although the procedure both reproduced results of previous researchers on low order test problems and produced plausible results where the DOA is not known a priori, difficulties with the performance of the inner loop optimization procedure were encountered on test problems, and the higher order gas jet system, which incorporate "hard" nonlinearities. As a result it was decided to postpone computational experience with the reaction wheel system -- which involves several "hard" nonlinearities -- until a deeper understanding of these problems has been obtained.
The outstanding research thus remains the refinement of the DOA estimation procedure -- in particular the inner loop Powe11-Han constrained optimization method or a suitable alternative.

Directions for further research. Difficulties with the Powell-Han algorithm similar to those encountered at the inner optimization loop of DOMATT have been experienced by other researchers, Lemarechal (1979a), Madsen (1979). Two identified sources of deficiency with the best current implementation of the algorithm (Harwell VA02AD as used in DOMATT) are relevant in the present context. The first deficiency is a tendency to sinqularity in the Lagrangian inverse Hessian update when steep-walled functions such as "hard" nonlinearities are encountered. The practical result is an attempt at a large algorithm step outside prescribed bounds for the state variables. The second shortcoming involves prematurely smazl algorithm steps on problems -- such as those posed by the attitude control systems-having highly curved constraint surfaces. The difficulties result from using Han's theoretical results on Lagrangian augmentation and quadratic approximation 1 ine search. These suggestions are directed at improvement of the present inner loop local optimization technique, but it is possible that other procedures for the local numerical solution of difficult nonlinear programming problems will have to be tried.

The DOMATT code includes inner loop starting point selection procedures designed to overcome the problem of selecting a local rather than the global optimum by the inner loop algorithm, and also for preventing the slow convergence at the outer loop caused by multiple global optima -- a problem identified by Shields and Storey (1975). These procedures can be refined by further application of combinatorial techniques. The precise nature of the starting point procedures selected depends on how closely the candidate inner loop solutions need to be tracked in successive iterations of the outer loop. This is likely to be fairly problem specific, so that detajled mathematical investigation of the $\vec{V}=0$ manifold could improve the efficiency of the DOMATT code. An important part of the future study is thus an investigation of how tangency points (local optima) found in the inner loop move as the $P$ matrix (quadratic Lyapunov function kernel) is altered at the outer 10op. This is a form of parametric information about the solution of nonlinear programming problems under perturbations -an area of considerable research activity at the moment. Unfortunately, current perturbation analyses of nonlinear programmes, see e.g. Fiacco (1982), are not immediately applicable in that they assume too much smoothness in the problem functions and perturbation parameter dependencies. It would be useful to extend such general analyses to the case of programmes with only directionalzy differentiable problem 
functions, ef. Dempster \& Wets (1976), which arise frequently in aerospace engineering applications.

An investigation must also be made of steplength tuning and outer loop gradient provision for the alternative BFGS quasiNewton algorithm utilized in DOMATT. These provisions are designed to enhance the efficiency of the outer loop algorithm. Regarding the second point, due to the complex nonlinear, but analytic, formulae represented by (38), see Dempster et al (1979), Appendix I, the development of gradient information should be investigated through the use of automatic differentiation software which has recently become available, Robinson (1979). Gradient information would also require of course the results of the perturbation analysis of the inner loop problem called for above.

The above refinements of the DOMATT code are currently under development using the test problems. While their difficulty should not be minimized, it is our belief that probilems of the complexity described above are close to the edge of solvability with current optimization theory and methods.

More generally, it would be fruitful to study the extension of Weissenberger's piecewise quadratic Lyapunov function techniques for single actuators to the 3-dimensional actuators used on spacecraft. This would allow study of the gas jet chattering phenomenon. The use of piecewise linear Lyapunov functions (generated by linear programming techniques) might also be investigated, of. Rosenbrock (1962). Such a method would give more careful mapping of DOA boundaries than is possible with quadratic techniques and would allow the extension of the DOMATT code to nondifferentiable systems more general than the present aerospace engineering application.

\section{ACKNOWLEDGEMENTS}

The research described above was initially carried out under Contract No. 3665/78/NL/ AK(SC) for ESTEC of the European Space Acency by Oxford Systems Associates Limited. The Study Team, under the leadership of the first author, consisted of D.W. Clark, J.F. Miles, I. Slater, A.M. U1ph and C.H. Whittington. The second author was the ESTEC Technical Representative. All these individuals contributed materially to the project. This paper is based on Dempster (1980b) and Coupe (1980). It is a pleasure for the first author to acknowledge helpful discussions with C. Lemarechal, K. Madsen and S.M. Robinson and the partial support of IIASA where his earlier paper was written. The earlier paper was presented to the Second Symposium on Mathematical Programming With Data Perturbations, Washington, D.C., 1-2 May, 1980.

\section{REFERENCES}

Adby, P.R. and M.A.H. Dempster (1974). Introduction to Optimization Methods. Chapman \& Ha11, London.

Coupé, G.M. (1980). Assessment of the state-of-the-art for simultaneous three axis large angle attitude manoeuvres. ESTEC Working Paper No. 1260.

Crouch, P.E. et al (1980). An Appraisal of Iinear Analytic Systems Theory With Applications to Attitude Control. Study Final Report, ESTEC Contract No. 3771/78/ NL/AK. Warwick Control Theory Centre.

Davison, E.J. and E.M. Kurak (1971). A computational method for determining quadratic Lyanpunov functions for nonlinear systems. Automatica 7, 627-536.

Dempster, M.A.H. (1980a). Study on stability and control of satellite large angle manoeuvres: Executive summary. TR8002, Oxford Systems Associates Limited, Oxford.

Dempster, M.A.H. (1980b). Nested optimization in DOA estimation for nonlinear dynamical systems: Spacecraft large angle manoeurres. IIASA Working Paper No. WP $-80-179$.

Dempster, M.A.H., J.F. Miles and C.H. Whittington (1979). Study on the Stability and Control of Satellite Large Angle Manoeuvres. Study Final Report, ESTEC Contract No. 3665/78/NL/AK(SC). Oxford Systems Associates Limited, Oxford.

Dempster, M.A.H. and R.J-B. Wets (1976). On regularity conditions in constrained optimization. Discussion Paper 7608, Center for Operations Research and Econometrics, Université Catholique de Louvain.

Fiacco, A.V., ed. (1982). Proceedings of the Second Symposium on Mathematical Programing With Data Perturbations. Lecture Notes in Pure and Applied Maths. Marcel Dekker, New York.

Geiss, G.R., V.D. Cohen, R.D'heedene, D. Rothschild and A. Chomas (1971). An Algorithm for Liapunov Stability Analysis of Complex Nonlinear Systems with Application to the Orbiting Astronomical Observatory. NASA Contractor Report CR-1729. Grumann Aerospace Corporation, Bethpage, N.Y.

Hrastar, J. (1970). Attitude control of a space craft with a strapdown inertial reference system and on-board computer. NASA TN-D-5959.

Lasalle, J. and S. Lefschetz (1961). Stability by Lyapunov's Direct Method with Applications. Academic, New York.

Lemarechal, C. (1979a). Private communication.

Lemarechal, C. (1979b). Numerical experiments in nonsmooth optimization. To appear in: Progress in Nondifferentiable optimization. E. Nurminski, ed. IIASA Collaborative Proceedings Series, Laxenburg.

Madsen, K. (1979). Private communication. 
Mortensen, R.E. (1963). On systems for automatic control of the rotation of a rigid body. Electronics Research Lab. Report 63-23. U. of California, Berkeley. Also in J. App Z. Math. \& Mech. 32 (1965), 228.

Mortensen, R.E. (1968). A globally stable attitude regulator. Int. $J$. Control 8.2, 297-302.

Murnaghan, F.D. (1962). The Unitary and Rotation Groups. Lectures on Applied Maths. No. 3. Spartan Books, Washington, D.C.

Powel1, M.J.D. (1964). An efficient method for finding the minimum of a function of several variables without calculating derivatives. Computer $J .7,155-162$.

Powell, M.J.D. (1977). A fast algorithm for nonlinearly constrained optimization calculations. In: Numerical Analysis, Dundee 1977. G.A. Watson, ed. Lecture Notes in Math. No. 630. Springer, Berlin (1978), 144-157.

Powel1, M.J.D. (1978). Algorithms for nonlinear constraints that use Lagrangian functions. Math. Programming 14, $224-248$.

Robinson, S.M. (1979). Private communication.

Rodden, J.J. (1964). Numerical applications of Lyapunov stability theory. Joint Automatic Control Conference (Stanford) 1964. 261-268.
Rosenbrock, H.H. (1962). A Lyapunov function with applications to some nonlinear physical systems. Automatica 1, 31-53.

Rouche, N., P. Habets and M. Laloy (1977). Stability Theory by Lyapunov's Direct Method. Applied Mathematical Sciences 22. Springer-Verlag, New York.

Shields, D.N. (1973). Ph.D. Thesis. Department of Mathematics, Loughborough University of Technology.

Shields, D.N. and C. Storey (1975). The behavior of optimal Lyapunov functions. Int. J. Control 21.4, 561-573.

Smith, P.G. (1971). Numerical solution of the matrix equation $\mathrm{A}^{\prime} \mathrm{X}+\mathrm{X} \mathrm{A}+\mathrm{B}=0$. IEEE Trans. Automatic Control. AC-16.3, 198-201.

Weissenberger, S. (1965). Stability Analysis of relay control systems via the direct method of Lyapunov. NASA CR-320. Washington.

Weissenberger, S. (1969). Piecewise-quadratic and piecewise-linear Lyapunov functions for discontinuous systems. Int. J. Control. 10.2, 171-180.

Zubov, V.I. (1955). Questions in the theory of Lyapunov's second method: The construction of the general solution in the domain of asymptotic stability. Prikz. Mat. I. Mekhan. 19, 197-210. (In Russian.) 


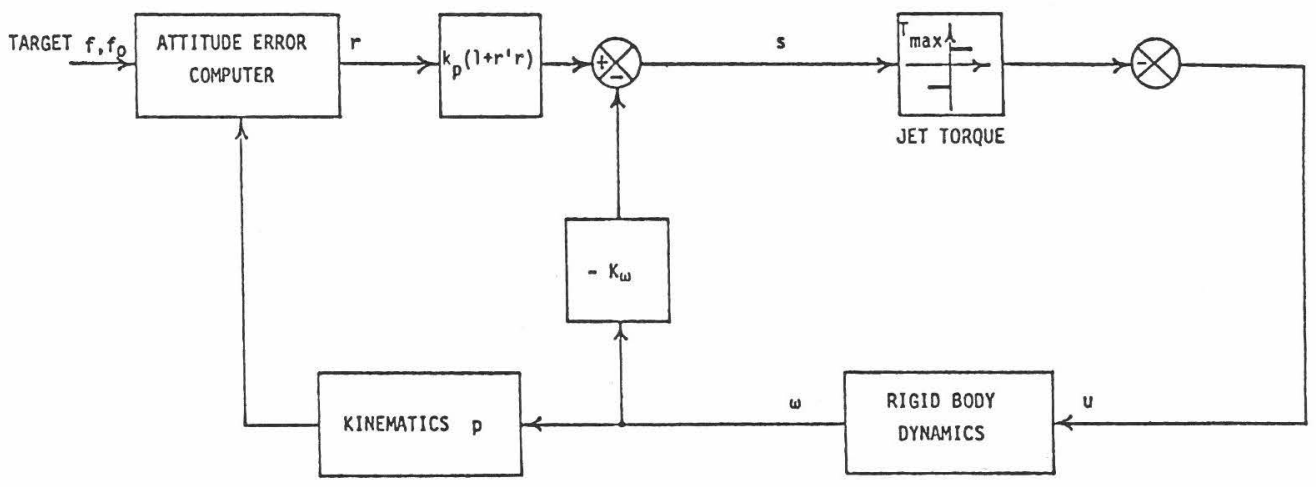

Fig. 1 Satellite Large Angle Att1tude Control System Reaction Jet Actuators

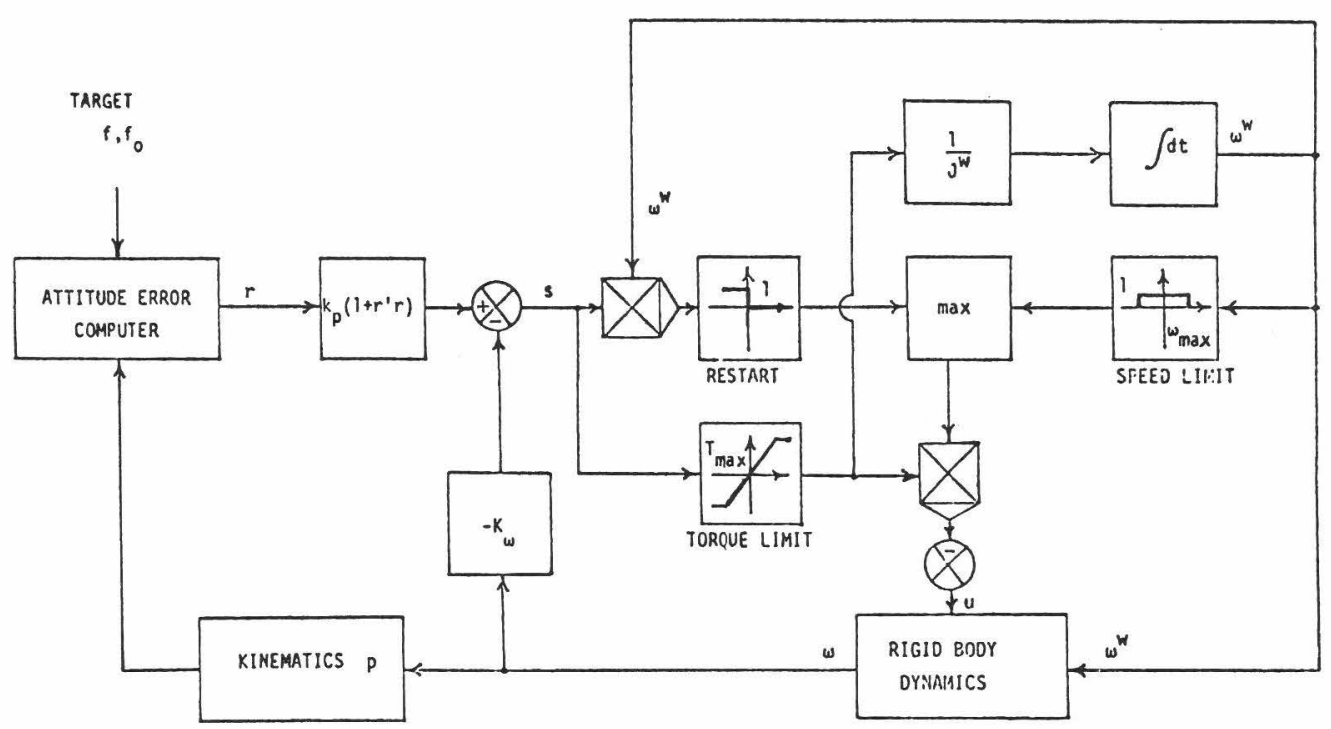

Fig. 2 Satellite Large Angle Attitude Control System Reaction Whecl Actuators 

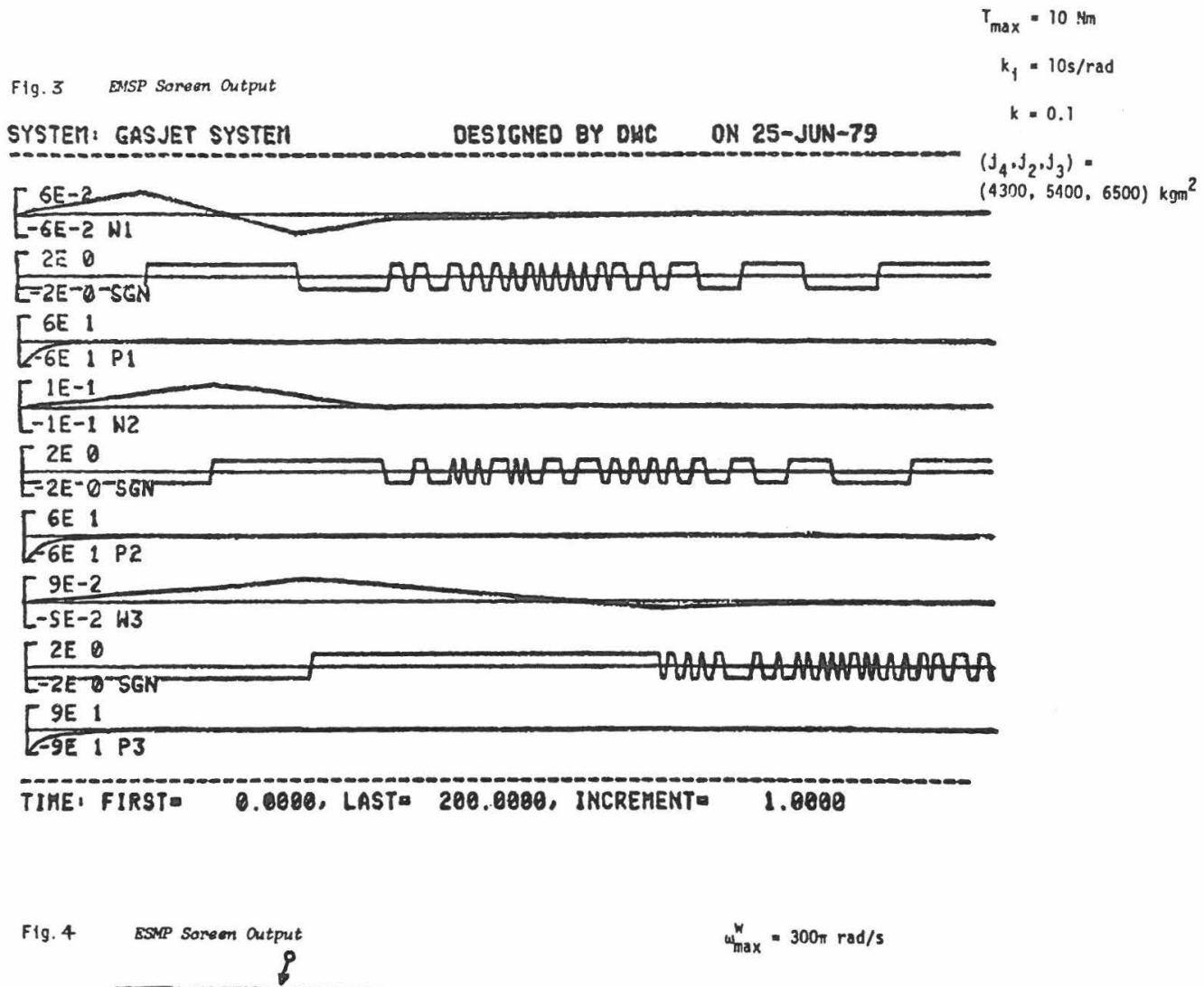

SYSTEM! REACTION H=10B KI=70 OESIGNEO BY DNC ON 18-JLL-79

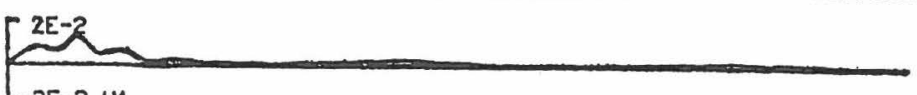

$[-2 E-2$ WI

CE 1

-6E 1 PI

$\overbrace{-7 E V_{3}}^{7 E-3}$

6E 1

$\left[\begin{array}{lll}-6 E & 1 & P 2\end{array}\right.$

$\left[\frac{7 E-3}{-7 E-3 / 6 / 3}\right.$

SE 1

L-9E 1 P3

TIME, FIRST $=0.0000$, LAST $=3000.0000$, INCREMENT 10.0000 


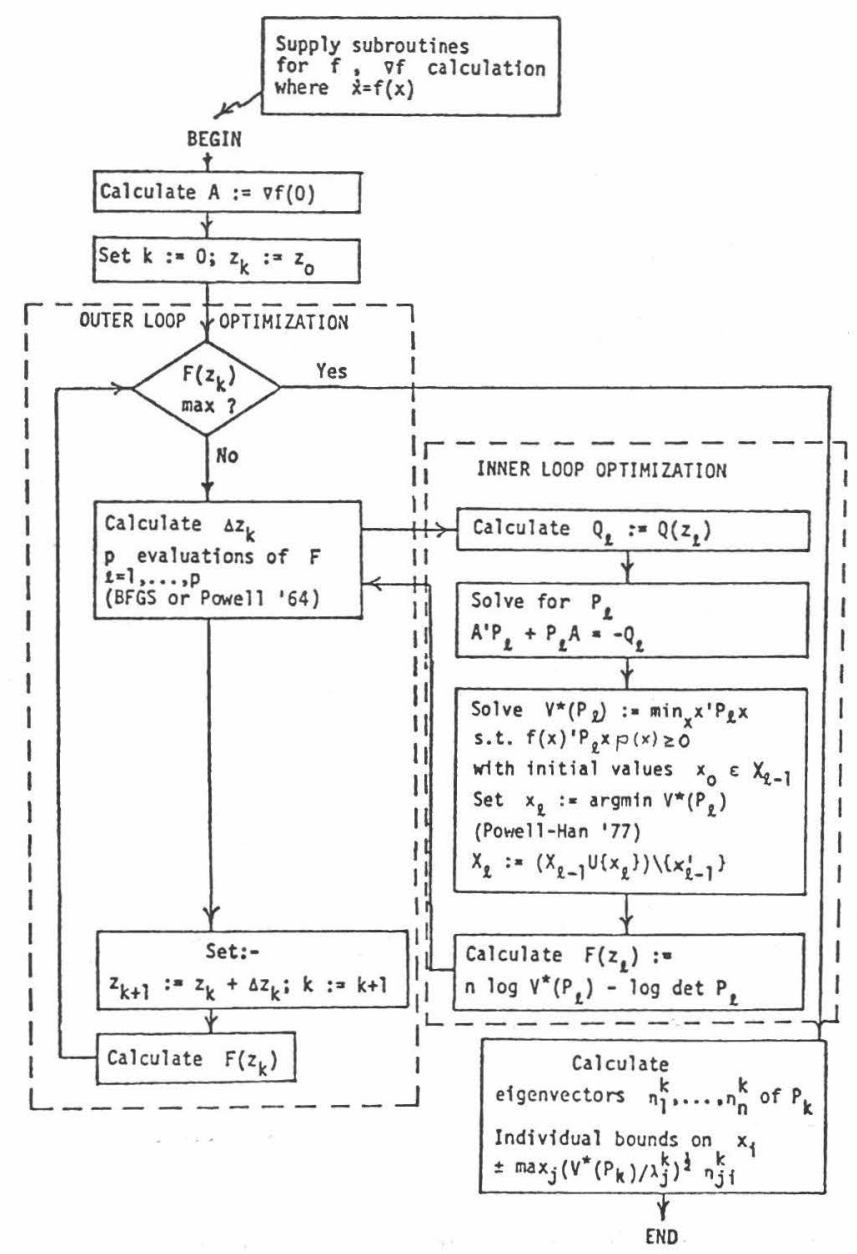

Fig 5 Flowchart of the Basic DOA Sstimation Algorithen 
